\section{Low prevalence of Salmonella enterica in cull dairy cattle at slaughter in Northern Italy}

\author{
Silvia Bonardi, ${ }^{1}$ Ilaria Bruini, ${ }^{1}$ \\ Rossella Magnani, ${ }^{2}$ Nicolò Cannistrà, ${ }^{2}$ \\ Franco Brindani ${ }^{1}$
}

${ }^{1}$ Food Hygiene Unit, Department of Veterinary Science, University of Parma, Parma; ${ }^{2}$ Parma Local Health Unit, National Veterinary Service, Parma, Italy

\begin{abstract}
In order to evaluate Salmonella carrier status of cull dairy cattle at slaughter, 125 animals were randomly selected during the period February-May 2016. Dairy cows were reared in 89 farms located in two regions of Northern Italy (Lombardy and Emilia-Romagna regions), where bovine milk is primarily used for ParmigianoReggiano cheese and Grana Padano cheese production. Samples were collected by swabbing a $400-\mathrm{cm}^{2}$ area of the brisket hide and by rectoanal mucosal swabs. They were tested following the reference ISO 6579 method and the isolates were serotyped following the Kauffmann-White-Le Minor scheme and genotyped by $X b a \mathrm{I}$ PFGE. Salmonella was detected in $1.6 \%$ of the brisket hide samples (2/125) $(95 \%$ CI: 0.45.6) and never found in faecal samples (95\% CI: $0-3 \%$ ). The positive cattle were reared in two farms located only in EmiliaRomagna region. The isolates were typed as $S$. Derby and $S$. Seftenberg. The comparison of the pulsed-field gel electrophoresis (PFGE) patterns of the bovine strains with all the PFGE patterns of the same serotypes responsible for human salmonellosis cases notified in Emilia-Romagna region in the years 2013-2015 did not find any correspondence. Therefore, the role of cull dairy cattle in transmitting Salmonella to humans seems to be less important than those of pigs and poultry in EU, but more data are needed for completing attribution source studies.
\end{abstract}

\section{Introduction}

Salmonella is an important zoonotic pathogen, often reported with high prevalence in animal production. It is the second most common foodborne pathogen in the European Union, with 88,715 confirmed laboratory cases reported in 2014. Human foodborne salmonellosis may be frequently acquired by eating raw or undercooked food of animal origin, as eggs, meat, milk and products thereof (EFSA and ECDC, 2015a).

To date, some studies on attributing foodborne salmonellosis in humans to animal reservoirs are based on the prevalence of Salmonella in animals and food from the EU-wide Baseline Studies conducted in the years 2006-2008 and from the European Union Summary Reports published by the European Food Safety Authority (EFSA) from 2006 to 2009, while data from human cases of salmonellosis are collected from The European Surveillance System (TESSy) administered by the European Centre for Disease Prevention and Control (ECDC) (De Knegt et al, 2015a). These attribution estimates suggest that layer hens were the most important source of salmonellosis in the EU in the study period, followed by pigs. Turkeys and broilers were found to be particularly important only in a few countries, as Denmark and Portugal respectively (De Knegt et al., 2015b).

These studies suggest that the role of cattle in transmitting Salmonella infection to humans by consumption of contaminated food is less important than other animal species. Nevertheless, if compared to pig and poultry data, cattle data collected by EU countries were in general poor and sometimes referred to clinical isolates only (De Knegt et al., 2015a), thus suggesting that more efforts are needed to monitor Salmonella contamination of food of bovine origin. For example, the prevalence of Salmonella-positive bovine carcasses reported by 13 EU Member States was $0.23 \%$ in 2014. In the same year, 225 Salmonella strong-evidence outbreaks were reported; pig meat and products thereof accounted for $9.3 \%$ outbreaks, broiler meat and products thereof for $3.6 \%$, bovine meat and products thereof for $2.2 \%$. Another category of meat, defined as meat and meat products accounted for $3.1 \%$ of the strongevidence outbreaks (EFSA and ECDC, 2015a). To study the role of cattle in human foodborne salmonellosis, raw milk at farm and carcasses at slaughter should be more frequently investigated. As verocytotoxinproducing Escherichia coli (VTEC) contamination of bovine carcasses at slaughter may occur via the faecal route or contaminated hide (EFSA, 2007), the same could occur also for Salmonella contamination (Narváez-Bravo et al., 2013). Salmonellacarrier cattle may end up as contaminated carcasses at slaughter or may contribute to cross-contamination of other carcasses during processing.

The main aim of the study was to investigate the prevalence of Salmonella carriers among cattle at slaughter in Northern Italy
Correspondence: Silvia Bonardi, Department of Veterinary Science, University of Parma, via del Taglio 10, 43126, Parma, Italy.

Tel: +39.0521.032744

E-mail: silvia.bonardi@unipr.it

Key words: Salmonella enterica; Cattle; Hide; Faeces; Slaughterhouse.

Acknowledgments: the authors gratefully acknowledge Dr. Gisella Pizzin, Ms. Ida Poli and Ms. Giuseppina Trentadue (Department of Veterinary Science, University of Parma, Italy) for technical assistance and Dr. Erika Scaltriti (Institute for Experimental Veterinary Medicine of Lombardy and Emilia Romagna Region, Parma, Italy) for serotyping and PFGE typing of the isolates.

Conflict of interest: the authors declared no potential conflict of interest.

Funding: the study was funded by the University of Parma, Italy.

Received for publication: 18 July 2016

Revision received: 18 November 2016.

Accepted for publication: 19 November 2016

This work is licensed under a Creative Commons Attribution-NonCommercial 4.0 International License (CC BY-NC 4.0).

CC Copyright S. Bonardi et al., 2017

Licensee PAGEPress, Italy

Italian Journal of Food Safety 2017; 6:6172

doi:10.4081/ijfs.2017.6172

and to compare the proportion of positive animals by testing cattle hide and faecal matter. The objective was also to investigate if the Salmonella isolates detected in cattle were responsible of notified human salmonellosis cases in the same geographic area (Emilia-Romagna region) of Northern Italy.

\section{Materials and Methods}

\section{Sampling}

From February to May 2016, a total of 125 cull dairy cattle at slaughter was randomly selected to be tested for Salmonella. Sampling collection was performed during eight visits, spaced one to two weeks. The number of animals tested per visit ranged from 10 to 20 (average number: 15.6). Dairy cattle were reared in 89 farms located in the provinces of Cremona, Mantua, Parma, Piacenza and Reggio Emilia (Lombardy and Emilia-Romagna regions, of Northern Italy). In those provinces bovine milk is primarily used for hard cheese production, as Parmesan cheese and Grana Padano cheese. Cattle were tested by swabbing a $400 \mathrm{~cm}^{2}$ 
area of the brisket hide, which is considered at risk because the brisket area of the hide is cut during dehiding and is thus a likely source of carcass contamination (EFSA, 2007). Swabbing was performed by using sterile sponges moistened with Buffered Peptone Water (BPW) (3M Health Care, S. Paul, MN, USA). Faecal samples were collected by using sterile cotton-tipped swabs and swabbing an area (3 to $5 \mathrm{~cm}$ ) inside the anal canal of each animal prior to slaughter. This kind of sampling is called rectoanal mucosal swabs (RAMS) and was used in similar studies (Agga et al., 2016; Beach et al., 2002). Swabs were immediately placed in sterile tubes containing $1 \mathrm{~mL}$ of Buffered Peptone Water (BPW; Oxoid, Basingstoke, UK). All samples were refrigerated after collection, transported to the laboratory within two hours and analysed on the same day. Confidence intervals in the observed prevalence were estimated by using the Wilson binomial approximation (Brown et al., 2001).

\section{Salmonella detection and typing}

A total of 250 samples (125 hide samples and 125 faecal samples) were tested following the ISO 6579:2002 method (hide samples) and the ISO 6579:2007 Amd.1 (faecal samples) (ISO, 2002, 2007). All samples were previously pre-enriched in BPW suspending each hide sponge in 90 $\mathrm{mL}$ and each RAMS sample in $9 \mathrm{~mL}$ of the liquid medium. The sponge suspensions were manually shaken for $2 \mathrm{~min}$. The cultures were then incubated at $37 \pm 1{ }^{\circ} \mathrm{C}$ for $18 \pm 2$ h. Following the ISO 6579:2002 method, after incubation the sponge cultures were inoculated into $10 \mathrm{~mL}$ of Mueller-Kauffmann tetrathionate broth (MKTT; Oxoid) and $10 \mathrm{~mL}$ of RappaportVassiliadis soy broth (RVSB; Oxoid), with dilution rations of $1: 10$ and $1: 100$, respectively. The MKTT and RVSB cultures were incubated at $37 \pm 1^{\circ} \mathrm{C}$ and $41.5 \pm 1^{\circ} \mathrm{C}$ for $24 \mathrm{~h}$, respectively. After incubation, $10-\mu \mathrm{L}$ of each culture was streaked onto Xylose Lysine Desoxycholate agar (XLD; Oxoid) and Chromogenic Salmonella agar (Oxoid) plates, incubated at $37 \pm 1^{\circ} \mathrm{C}$ for $24 \mathrm{~h}$. Suspect colonies were seeded into Triple Sugar Iron Agar (Biolife, Milan, Italy), Lysine Iron Agar (Oxoid) and Christensen's Urea Agar (Biolife) and incubated at $37 \pm 1^{\circ} \mathrm{C}$ for $20-24 \mathrm{~h}$. Typical Salmonella colonies were tested by slide agglutination with an O-omnivalent Salmonella serum (Denka Seiken, Tokyo, Japan). Biochemical identification to the genus level was performed by using the API $20 \mathrm{E}^{\circledR}$ microsubstrate system (bioMérieux, Marcy 1'Etoile, France). Following the ISO 6579:2007 Amd. 1 method, after incubation $100-\mu \mathrm{L}$ of each faecal culture in BPW was seeded onto plates of Modified semisolid Rappaport Vassiliadis (MSRV; Oxoid) following ISO recommendations (3 drops placed separately on the plate surface). After incubation at $41.5 \pm 1^{\circ} \mathrm{C}$ for $24 \mathrm{~h} \pm 3 \mathrm{~h}$, the plates showing a grey-white zone extending out of the inoculum were further tested by streaking a $10-\mu \mathrm{L}$ loopful of the bacterial growth onto XLD (Oxoid) agar plates. Plates not showing bacterial growth were incubated for further $24 \pm 3 \mathrm{~h}$, after that they were considered negative if no grey-white growth has developed. XLD agar plates were incubated at $37 \pm 1^{\circ} \mathrm{C}$ for $24 \mathrm{~h}$ and suspect Salmonella colonies were selected and subjected to ISO 6579 confirmation tests as previously described. The reference Salmonella strain ATCC 14028 was used as positive controls.

Salmonella serotyping was performed according to the White-Kauffmann-Le Minor scheme by slide agglutination with $\mathrm{O}$ and $\mathrm{H}$ antigen specific sera (Bio-Rad, Marnes-La Coquette, France; Denka Seiken, Tokyo, Japan; Sifin, Berlin, Germany). Salmonella pulsed-field gel electrophoresis (PFGE) typing was performed according to standard methods (PulseNet, 2010) with $\mathrm{XbaI}$ restriction of DNA.

\section{Results}

Hide sponges: Salmonella was detected in $1.6 \%$ of the brisket hide samples $(2 / 125)$ (95\% CI: 0.4-5.6). The positive animals were reared in two-arms located in EmiliaRomagna region. The two isolates were serotyped as $S$. Dublin and $S$. Senftenberg. The comparison of the PFGE patterns of the bovine strains to all the PFGE patterns of the same serotypes that were identified among human salmonellosis cases notified in Emilia-Romagna region in the years 2013-2015, did not find any correspondence. The human Salmonella isolates were sero-typed and PFGE-typed within the regular food-borne diseases surveillance activity by the Institute for Experimental Veterinary Medicine of Lombardy and Emilia-Romagna (Italy). The comparison of the pulsotypes of the two isolates of $S$. Dublin and $S$. Seftenberg with the PFGE profiles of the same serovars detected in cattle during the period 2011-2015, demonstrated that the PFGE type of $S$. Dublin detected in the present study was rather common among bovine population in Italy, while the pulsotype of $S$. Senftenberg was not frequently identified. RAMS samples: Salmonella was never detected in the faecal samples tested (95\% CI: 0-3\%).

\section{Discussion}

In the present study, Salmonella was detected from hide samples only thus confirming the role of cattle hide as likely source of Salmonella onto carcasses during slaughtering. Other studies support the role of hide in beef contamination at slaughter, even if a wide variation in prevalence has been reported. It ranged from $36.25 \%$ in Venezuela (Narváez-Bravo et al., 2013), $15.4 \%$ in the USA (Bacon et al., 2002) to $0.75 \%$ in Ireland (Khen et al., 2014). Very high prevalence values were reported by Brichta-Harhay et al. (2008), which detected Salmonella in $89.6 \%$ of cull cattle hides in the USA, and by Fegan et al. (2005) which found $68 \%$ hide prevalence in Australia. These differences can be attributable to epidemiological factors, breeding conditions, management practices, transportation and lairage hygiene, analytical methods or sampling strategies.

In our study, all faecal samples were negative for Salmonella. This result can be attributable to different factors: i) Salmonella shedding by cattle is commonly intermittent (Wray and Sojka, 1977) and therefore faecal samples can test negative at slaughter; ii) hide contamination could have been previously acquired at farm, during transportation or at lairage from other shedding cattle or contaminated environment. As shown in a study by Beach et al. (2002), hide contamination with Salmonella increased in adult cattle prior and after transport from farm to slaughter from $20 \%$ to $56 \%$ and thus contaminated trucks and holding pens could play a role in Salmonella transmission to hides before slaughter. Regarding the use of RAMS instead of manual collection of faeces from the rectum (faecal grab; FG), RAMS is considered a more practical and reliable alternative to FG for assessing Salmonella fecal shedding status among cattle (Agga et al., 2016).

Considering that the prevalence on cull cows hides was very low, the negativity of rectal samples was a reliable finding. As a matter of fact, in other studies Salmonella prevalence on cattle hides was higher than in faeces as shown by Fegan et al. (2005), which detected $16 \%$ of positive faeces $v s$ $68 \%$ of positive hides and by NarváezBravo et al. (2013), which found $13.75 \%$ of positive faeces $v s 36.25 \%$ of positive hides.

In our study, the isolates detected on hides belonged to serovars frequently found in cattle. Infections by $S$. enterica subsp. enterica serovar Dublin ( $S$. Dublin) is of concern in cattle industries in several countries because of economic losses and health injuries as high mortality, abortion and 
reduction in milk production (Carrique-Mas et al., 2010; Lewerin et al., 2011). In cattle, Dublin was the second most frequently detected serovar (29.4\%) after Typhimurium (38.6\%) in 2013 at EU level (EFSA and ECDC, 2015b). S. Dublin may be persistent in dairy farms, with an average duration of infection of approximately 2 years (Nielsen and Dohoo, 2013) due to its environmental survival (Findlay, 1972) and persistent infection in some animals (House et al., 1993). Uncommon but serious human infections are reported and are mainly attributed to the consumption of raw or undercooked beef or unpasteurised milk products (Helms et al., 2003; Maguire et al., 1992). As expected, $S$. Dublin is frequently reported among serovars detected in bovine meat in the European countries (EFSA and ECDC, 2015b).

$S$. enterica subsp. enterica serovar Senftenberg ( $S$. Senftenberg) is not frequently reported from cattle, but in some studies conducted in the USA it was one of the most prevalent serovars (Fitzgerald et al., 2003). Noteworthy, if $S$. Dublin is closely related to cattle, $S$. Senftenberg can be transmitted to humans by a variety of foods, including poultry (Pedersen et al., 2008) and vegetables like basil and pistachios (CDC, 2016; Pezzoli et al., 2008). The isolation from cattle at slaughter in Italy strengthen the hypotheses that $S$. Senftenberg could survive on cattle hide and be transmitted from bovines to the slaughterhouse environment and, potentially, to beef carcasses.

\section{Conclusions}

In this study a very low prevalence of Salmonella carriers among cull dairy cows at slaughter was detected. Nevertheless, since beef may be responsible for transmission of Salmonella to consumers and outbreaks from raw or undercooked beef products (as roast-beef and ground beef) can occur (Friesema et al., 2012; Laufer et al., 2016), bacteriological monitoring of cattle presented at slaughter is important for both epidemiological and attribution estimates. A recent attribution source study of human salmonellosis suggested that the median percent probabilities for human cases to originate from cattle ranged from 3 to $7 \%$ according to age groups ( $7 \%$ in children 5 17 years old) and from 2 to $6 \%$ according to urbanisation degree (6\% in rural areas). According to season, in autumn and winter (October-March) 7\% of the cases could occur vs $4 \%$ of the cases in spring and summer (April-September) (Mughini-Gras et al., 2014). In our country, the interest in the epidemiological role of cattle is supported by the paucity of data on Salmonella in cattle at slaughter. However, to enable more detailed conclusions regarding contamination routes of Salmonella at slaughter, future studies involving higher numbers of cattle are needed. The present study can be thus considered a preliminary research to investigate the role of cull dairy cows as likely source of Salmonella to beef during slaughter operations.

\section{References}

Agga GE, Arthur TM, Schmidt JW, Wang R, Brichta-Harhay DM, 2016. Diagnostic accuracy of rectoanal mucosal swab of feedlot cattle for detection and enumeration of Salmonella enterica. J Food Protect 79:531-7.

Bacon RT, Sofos JN, Belk KE, Hyatt DR, Smith GC, 2002. Prevalence and antibiotic susceptibility of Salmonella isolated from beef animal hides and carcasses. J Food Protect 65:284-90.

Beach JC, Murano EA, Acuff GR, 2002. Prevalence of Salmonella and Campylobacter in beef cattle from transport to slaughter. J Food Protect 65:1687-93.

Brichta-Harhay DM, Guerini MN, Arthur TM, Bosilevac JM, Kalchayanand N, Shackelford SD, Wheeler TL, Koohmaraie M, 2008. Salmonella and Escherichia coli O157:H7 contamination on hides and carcasses of cull cattle presented for slaughter in the United States: an evaluation of prevalence and bacterial loads by immunomagnetic separation and direct plating methods. Appl Environ Microbiol 74:6289-97.

Brown LD, Cat TT, DasGupta A, 2001. Interval estimation for a binomial proportion. Stat Sci 16:101-33.

Carrique-Mas JJ, Willmington JA, Papadopoulou C, Watson EN, Davies $\mathrm{RH}, 2010$. Salmonella infection in cattle in Great Britain, 2003 to 2008. Vet Rec 167:560-5.

CDC, 2016. Multistate outbreak of Salmonella Montevideo and Salmonella Senftenberg infections linked to Wonderful Pistachios (final update). Centers for Disease Control and Prevention www.cdc.gov/salmonella/montevideo-03-16/

De Knegt LV, Pires SM, Hald T, 2015a. Using surveillance and monitoring data of different origins in a Salmonella source attribution model: a European Union example with challenges and proposal solutions. Epidemiol Infect
143:1148-65.

De Knegt LV, Pires SM, Hald T, 2015b. Attributing foodborne salmonellosis in humans to animal reservoirs in the European Union using a multi-country stochastic model. Epidemiol Infect 143:1175-86.

EFSA and ECDC, 2015a. The European Union summary report on trends of zoonoses, zoonotic agents and foodborne outbreaks in 2011. EFSA J 13:4329.

EFSA and ECDC, 2015b. The European Union summary report on trends of zoonoses, zoonotic agents and foodborne outbreaks in 2013. EFSA J 13:3991.

EFSA, 2007. Monitoring of verotoxigenic Escherichia coli (VTEC) and identification of human pathogenic VTEC types. EFSA J 579:1-61.

Fegan N, Vanderlinde P, Higgs G, Desmarchelier P, 2005. A study of the prevalence and enumeration of Salmonella enterica in cattle and on carcasses during processing. J Food Protect 68: 1147-53.

Findlay CR, 1972. The persistence of Salmonella Dublin in slurry in tanksand on pasture. Vet Rec 91:233-5.

Fitzgerald AC, Edrington TS, Looper ML, Callaway TR, Genovese KJ, Bischoff KM, McReynolds JL, Thomas JD, Anderson RC, Nisbet DJ, 2003. Antimicrobial susceptibility and factors affecting the shedding of E. coli O157:H7 and Salmonella in dairy cattle. Lett Appl Microbiol 37:392-8.

Friesema IHM, Schimmer B, Ros JA, Ober HJ, Heck MEOC, Swaan CM, de Jager CM, Peran i Sala RM, van Pelt W, 2012. A regional Salmonella enterica serovar Typhimurium outbreak associated with raw beef products, The Netherlands, 2010. Foodborne Pathog Dis 9:102-7.

Khen BK, Lynch OA, Carroll J, McDowell DA, Duffy G, 2014. Prevalence and characteristics of Salmonella in the beef chain in the Republic of Ireland. Zoonoses Public Health 61:534-6.

Helms M , Vastrup P , Gerner-Smidt P , Mølbak K , 2003. Short and long term mortality associated with foodborne bacterial gastrointestinal infections: registry based study. Brit Med J 326:357-61.

House JK, Smith BP, Dilling GW, Roden LD, 1993. Enzyme-linked immunosorbent assay for serologic detection of Salmonella Dublin on a large dairy. Am J Vet Res 54:1391-9.

ISO, 2002. Microbiology of food and animal feeding stuffs. Horizontal method for the detection of Salmonella spp. ISO 
Norm 6579:2002. International Standardization Organization ed., Geneve, Switzerland.

ISO, 2007. Microbiology of food and animal feeding stuffs. Horizontal method for the detection of Salmonella spp. ISO Norm 6579:2002. Amendment 1: Annex D: Detection of Salmonella spp. in animal faeces and in environmental samples from the primary production stage. ISO Norm 6579:2007 Amd.1. International Standardization Organization ed., Geneve, Switzerland.

Laufer AS, Grass J, Holt K, Whichard JM, Griffin PM, Gould LH, 2016. Outbreaks of Salmonella infections attributed to beef - United States, 19732011. Epidemiol Infect 143:2003-13.

Lewerin SS, Skog L, Frössling J, Wahlström H, 2011. Geographical distribution of Salmonella infected pig, cattle and sheep herds in Sweden 19932010. Acta Vet Scand 53:51-8.

Maguire H, Cowden J, Jacob M, Rowe B, Roberts D, Bruce J, Mitchell E, 1992.
An outbreak of Salmonella Dublin infection in England and Wales associated with a soft unpasteurized cows' milk cheese. Epidemiol Infect 109:38996.

Mughini-Gras L, Enserink R, Friesema I, Heck M, van Duynhoven Y, van Pelt W, 2014. Risk factors for human salmonellosis originating from pigs, cattle, broiler chickens and egg laying hens: a combined case-control and source attribution analysis. PLoS One 9:e87933.

Narváez-Bravo C, Rodas-González A, Fuenmayor Y, Flores-Rondon C, Carruyo G, Moreno M, Perozo-Mena A, Hoet AE, 2013. Salmonella on feces, hides and carcasses in beef slaughter facilities in Venezuela. Int $\mathrm{J}$ Food Microbiol 166:226-30.

Nielsen LR, Dohoo I, 2013. Time-to-event analysis of predictors for recovery from Salmonella Dublin infection in Danish dairy herds between 2002 and 2012 . Prev Vet Med 110:370-8.

Pedersen TB, Olsen JE, Bisgaard M, 2008.
Persistence of Salmonella Senftenberg in poultry production environments and investigation of its resistance to desiccation. Avian Pathol 37:421-7.

Pezzoli L, Elson R, Little CL, Yip H, Fisher I, Yishai R, Anis E, Valinsky L, Biggerstaff $M$, Patel N, Mather $\mathrm{H}$, Brown DJ, Coia JE, van Pelt W, Nielsen EM, Ethelberg S, de Pinna E, Hampton MD, Peters T, Threlfall J, 2008. Packed with Salmonella-investigation of an international outbreak of Salmonella Senftenberg infection linked to contamination of prepacked basil in 2007. Foodborne Pathog Dis 5:661-8.

PulseNet, 2010. One-day (24-28 h) standardized laboratory protocol for molecular subtyping of Escherichia coli O157:H7, Salmonella serotypes, Shigella sonnei, and Shigella flexneri by Pulsed Field Gel Electrophoresis (PFGE). PulseNet Int 2010:1-16.

Wray C, Sojka WJ, 1977. Reviews of the progress of dairy science: bovine salmonellosis. J Dairy Res 44:383-425. 